\title{
Estabilidade dos potenciais evocados auditivos em indivíduos adultos com audição normal
}

\author{
Stability of auditory evoked potentials in adults \\ with normal hearing
}

\author{
Carla Gentile Matas, Natália Moribe Hataiama, Isabela Crivellaro Gonçalves
}

\begin{abstract}
RESUMO
Objetivo: Avaliar a estabilidade dos parâmetros dos potenciais evocados auditivos em adultos normais. Métodos: Foram submetidos à avaliação audiológica e eletrofisiológica (potencial evocado auditivo de tronco encefálico - PEATE, potencial evocado auditivo de média latência - PEAML e potencial cognitivo - P300) 49 indivíduos normais, de 18 a 40 anos (25 do gênero feminino e 24 do gênero masculino). Realizou-se reavaliação três meses após a avaliação. Resultados: Foram observadas diferenças entre os gêneros na avaliação para as latências das ondas III e V e interpicos I-III e I-V do PEATE e amplitude N2-P3 do P300. Não foram verificadas diferenças significativas para os parâmetros do PEATE, PEAML (latência das ondas Na, Pa e amplitude Na - Pa) e P300 (latência da onda P300) entre os resultados obtidos na avaliação e reavaliação. Conclusão: Exceção feita à amplitude N2-P3, observou-se estabilidade dos parâmetros de PEATE, PEAML e P300 em adultos normais após período de três meses.
\end{abstract}

Descritores: Testes auditivos; Audição; Potenciais evocados auditivos; Potencial evocado P300; Adulto

\section{INTRODUÇÃO}

Os Potenciais Evocados Auditivos (PEA) têm como objetivo avaliar a atividade neuroelétrica na via auditiva desde o nervo auditivo até o córtex cerebral ${ }^{(1)}$. A larga utilização na prática clínica fonoaudiológica torna-se importante a obtenção de dados acerca de sua confiabilidade no teste-reteste.

Os PEA têm se caracterizado como importante ferramenta em neurociência, pelo seu caráter objetivo na avaliação da integridade estrutural e funcional do sistema nervoso auditivo central. Além das reconhecidas aplicações clínicas dos PEA no diagnóstico audiológico, monitoramento intra-operatório e da função cognitiva, sua utilização possui vantagens no exame de casos de distúrbios de linguagem, por não necessitar de

Trabalho realizado no Laboratório de Investigação Fonoaudiológica em Potenciais Evocados Auditivos do Curso de Fonoaudiologia da Faculdade de Medicina, Universidade de São Paulo - USP - São Paulo (SP), Brasil, com bolsa concedida pela Fundação de Amparo à Pesquisa do Estado de São Paulo - FAPESP.

(1) Curso de Fonoaudiologia da Faculdade de Medicina, Universidade de São Paulo - USP - São Paulo (SP), Brasil.

(2) Curso de Fonoaudiologia da Faculdade de Medicina, Universidade de São Paulo - USP - São Paulo (SP), Brasil.

(3) Programa de Pós-graduação em Ciências da Reabilitação do Departamento de Fisioterapia, Fonoaudiologia e Terapia Ocupacional da Faculdade de Medicina, Universidade de São Paulo - USP - São Paulo (SP), Brasil. Endereço para correspondência: Carla Gentile Matas. R. Cipotânea, 51, Cidade Universitária, São Paulo (SP), Brasil, CEP: 05360-000. E-mail: cgmatas@usp.br

Recebido em: 22/3/2010; Aceito em: 28/6/2010 resposta verbal. A existência de associação entre alterações de linguagem oral ou escrita e disfunção nos processos de audição é descrita na literatura ${ }^{(2)}$. Em resumo, os PEA podem fornecer evidências eletrofisiológicas sobre o processamento auditivo da informação lingüística e, uma vez que são sensíveis às mudanças eletrofisiológicas após-estimulação, constituem medida objetiva acerca do monitoramento do processo terapêutico ${ }^{(3)}$.

Pesquisas com PEA relacionados a eventos, em avaliações de indivíduos de diferentes faixas etárias, em diferentes intervalos de tempos no teste-reteste, têm indicado que tais medidas são replicáveis e estáveis; daí a utilidade de estudos que incluem a comparação e seguimento de diferentes grupos ${ }^{(4-7)}$.

O objetivo deste estudo foi avaliar a estabilidade da latência e amplitude dos PEA de curta e média latências e potencial cognitivo em indivíduos adultos audiologicamente normais.

\section{MÉTODOS}

Este estudo foi aprovado pela Comissão de Ética para Análise de Projetos de Pesquisa (CAPPesq) do Hospital das Clínicas da Faculdade de Medicina da Universidade de São Paulo (HCFMUSP) sob n ${ }^{\circ}$ 0642/07.

Participaram 49 indivíduos na faixa etária de 18 a 40 anos, 25 do gênero feminino e 24 do masculino. Os indivíduos foram esclarecidos quanto aos procedimentos a serem realizados e assinaram consentimento para participar da pesquisa.

Os critérios de exclusão para composição da amostra foram: presença de perdas auditivas, distúrbio de processamento 
auditivo, déficits de linguagem, qualquer comprometimento clínico e/ou cognitivo que impedisse ou dificultasse a realização dos exames.

Os materiais e equipamentos utilizados na avaliação audiológica foram: otoscópio da marca Heine, para visualização do meato acústico externo; protocolo de coleta da história clínica; analisador de orelha média GSI-33 marca Grason-Stadler@ para realização de medidas de imitância acústica; audiômetro GSI-68 marca Grason-Stadler ${ }^{\circledR}$, e fone supra aurais TDH-50, para realização da audiometria tonal e logoaudiometria, em cabina acústica; listas de vocábulos ${ }^{(8)}$ para pesquisa do Limiar de Reconhecimento de Fala (LRF) e Índice Percentual de Reconhecimento de Fala (IPRF).

$\mathrm{Na}$ avaliação eletrofisiológica foram utilizados: equipamento portátil Traveler Express marca Bio-logic $₫$, quatro eletrodos de superfície, fones TDH-39. Foram utilizados procedimentos para garantir a segura e confiável captação dos potenciais.

Primeiramente, os indivíduos foram submetidos à anamnese, inspeção do meato acústico externo, audiometria tonal, logoaudiometria e medidas de imitância acústica, com o objetivo de selecionar os indivíduos com acuidade auditiva normal, com base nos critérios de normalidade adotados ${ }^{(8-12)}$.

Em seguida, realizou-se a avaliação eletrofisiológica da audição, por meio do PEATE, PEAML e P300 em ambiente protegido e isolado do ponto de vista elétrico e acústico.

No PEATE, o estímulo utilizado foi o clique de polaridade rarefeita, apresentado monoauralmente a $80 \mathrm{dBnNA}$, numa taxa de apresentação de 19,1 cliques por segundo com duração de 0,1 milissegundos (ms), perfazendo o total de 2000 estímulos. A janela de gravação foi de $10 \mathrm{~ms}$. Os filtros utilizados foram: passa-alto de $100 \mathrm{~Hz}$ e passa-baixo de $3 \mathrm{kHz}$. Foram obtidos dois traçados em cada orelha. Os eletrodos foram posicionados na fronte $(\mathrm{Fpz})$, nas orelhas direita e esquerda (A2 e A1). Foi considerado como eletrodo ativo, o fixado na mastóide da orelha testada, referência, o fixado na fronte e eletrodo terra o fixado na orelha contralateral da orelha testada.

No PEAML, o estímulo utilizado foi o clique, apresentado monoauralmente a $70 \mathrm{dBnNA}$, numa taxa de apresentação de 9,9 cliques por segundo, perfazendo o total de 1000 cliques. A janela de gravação foi de $100 \mathrm{~ms}$. Os filtros utilizados foram: passa-alto de $10 \mathrm{~Hz}$ e passa-baixo de $150 \mathrm{~Hz}$. Os eletrodos foram fixados na fronte (Fpz), A2, A1 e nas junções têmporoparietais direita e esquerda (C4 e C3). Foram considerados como eletrodos ativos os fixados nas mastóides, referência os fixados nas junções temporo-parietais, e eletrodo terra o fixado na fronte.

No P300, o estímulo utilizado foi o tone-burst em $1 \mathrm{e}$ $1,5 \mathrm{kHz}$, apresentado monoauralmente a $75 \mathrm{dBnNA}$, numa taxa de apresentação de 1,1 estímulos por segundo, num total de 300 estímulos. A janela de gravação foi de $512 \mathrm{~ms}$. Os filtros utilizados foram: passa-alto de $1 \mathrm{~Hz}$ e passa-baixo de $30 \mathrm{~Hz}$. Os eletrodos foram posicionados em Cz, A2, A1 e na fronte (Fpz), sendo considerado como ativo o eletrodo fixado na mastóide da orelha testada, referência o fixado em $\mathrm{Cz}$ e eletrodo terra o fixado na fronte. O estímulo raro ocorreu de 15 a $20 \%$ do total de estímulos e o indivíduo foi orientado a prestar a atenção e indicar o número de ocorrências $(1,5 \mathrm{kHz})$, em uma série de estímulos frequentes $(1 \mathrm{kHz})^{(13,14)}$.

Após os procedimentos preparatórios usuais, os eletrodos foram fixados à pele em posições pré-determinadas. Os valores de impedância dos eletrodos encontravam-se abaixo de cinco kOhms.

As variáveis analisadas em cada potencial evocado auditivo foram:

- PEATE: latências absolutas das ondas I, III e V e interpicos I-III, III-V e I-V (em ms);

- PEAML: latências das ondas $\mathrm{Na}$ e Pa (em ms) e amplitude $\mathrm{Na}-\mathrm{Pa}(\mathrm{em} \mu \mathrm{V})$ para cada modalidade - ipsilateral (C3/A1 e C4/A2) e contralateral (C3/A2 e C4/A1);

- P300: latência da onda P300 (em ms) e amplitude N2-P3 (em $\mu \mathrm{V})$.

Após o período de três meses, os indivíduos foram submetidos à reavaliação e tais resultados foram comparados com aqueles obtidos na avaliação inicial. Após esta comparação, calculou-se a diferença entre os resultados obtidos na avaliação e reavaliação para cada indivíduo. Os dados foram analisados quantitativamente, por meio de médias, medianas e desvios padrão para cada variável estudada. Para a análise estatística foi utilizado o teste $\mathrm{t}$ de Student. O nível de significância adotado foi de 0,05 (5\%). Os resultados dos potenciais evocados auditivos foram considerados estáveis quando não se observou diferenças entre os resultados obtidos na avaliação e reavaliação.

\section{RESULTADOS}

Para ambos os gêneros, não foram observadas diferenças entre as orelhas direita e esquerda no PEATE e P300. Então, para esses potenciais as análises foram baseadas nos valores obtidos em ambas as orelhas. Para o PEAML, as análises foram realizadas a partir dos valores obtidos em cada indivíduo, tendo em vista que sua interpretação é efetuada por modalidade (C3/ $\mathrm{A} 1, \mathrm{C} 3 / \mathrm{A} 2, \mathrm{C} 4 / \mathrm{A} 1$ e C4/A2).

Comparando-se os resultados do PEATE obtidos na avaliação e reavaliação, pode-se verificar que para os indivíduos dos gêneros feminino e masculino não houve diferença para as latências das ondas I, III e V ( $\mathrm{p}=0,95 ; 0,76 ; 0,94 ; 0,82 ; 0,37$ e 0,21 , respectivamente) e interpicos I-III, III-V e I-V ( $\mathrm{p}=0,81$; 0,$74 ; 0,85 ; 0,38 ; 0,12$ e 0,62 , respectivamente).

Nas Tabelas 1, 2 e 3 encontram-se os valores de média, mediana e desvio padrão referentes às diferenças obtidas a partir da comparação entre os resultados da avaliação e reavaliação de cada indivíduo, para as variáveis do PEATE, PEAML e P300, respectivamente.

Comparando-se os resultados do PEAML obtidos na avaliação e reavaliação, não se observou diferença para as latências da onda $\mathrm{Na}$ em todas as modalidades estudadas no gênero feminino $(\mathrm{p}=0,60 ; 0,33 ; 0,10$ e 0,74 , respectivamente C3/A1, C4/A1, C3/A2 e C4/A2) e masculino ( $\mathrm{p}=0,75 ; 0,72$; 0,58 e 0,60, respectivamente C3/A1, C4/A1, C3/A2 e C4/ A2). Para a latência da onda Pa, quando da comparação entre os resultados obtidos na avaliação e reavaliação, não houve diferença estatisticamente significante nos gêneros feminino $(\mathrm{p}=0,32 ; 0,16 ; 0,07$ e 0,14 , respectivamente C3/A1, C4/A1, 
C3/A2 e C4/A2), e masculino ( $\mathrm{p}=0,39 ; 0,16 ; 0,72$ e 0,25 , respectivamente C3/A1, C4/A1, C3/A2 e C4/A2). A amplitude

Tabela 1. Diferença entre os valores de latência das Ondas I, III e V e Interpicos I-III, III-V e I-V do PEATE obtidos na avaliação e reavaliação nos gêneros feminino e masculino

\begin{tabular}{cccccc}
\hline Gênero & $\begin{array}{c}\text { PEATE } \\
\text { (em ms })\end{array}$ & N & Média & Mediana & DP \\
\hline \multirow{4}{*}{ F I } & 50 & 0 & 0 & 0,09 \\
& III & 50 & 0 & 0 & 0,11 \\
& V & 50 & 0 & 0,02 & 0,14 \\
& I-III & 50 & 0 & 0 & 0,12 \\
& III-V & 50 & 0,01 & 0 & 0,14 \\
& I-V & 50 & 0 & 0 & 0,15 \\
\hline \multirow{4}{*}{ M } & I & 48 & 0 & 0 & 0,13 \\
& III & 48 & $-0,01$ & 0 & 0,11 \\
& V & 48 & 0,08 & 0 & 0,44 \\
& I-III & 48 & $-0,02$ & $-0,02$ & 0,15 \\
& III-V & 48 & 0,05 & 0,04 & 0,14 \\
& I-V & 48 & 0,01 & 0 & 0,16 \\
\hline
\end{tabular}

Legenda: $\mathrm{PEATE}=$ potencial evocado auditivo de tronco encefálico; $\mathrm{N}=$ número de orelhas; $\mathrm{M}$ = masculino; $\mathrm{F}$ = feminino; $\mathrm{ms}$ = milissegundos; $\mathrm{DP}=$ desvio-padrão
Na-Pa, tanto no gênero feminino ( $\mathrm{p}=0,34 ; 0,70 ; 0,12$ e 0,30 , respectivamente $\mathrm{C} 3 / \mathrm{A} 1, \mathrm{C} 4 / \mathrm{A} 1, \mathrm{C} 3 / \mathrm{A} 2$ e $\mathrm{C} 4 / \mathrm{A} 2)$ como no masculino ( $\mathrm{p}=0,84 ; 0,11 ; 0,53$ e 0,30 , respectivamente $\mathrm{C} 3$ / $\mathrm{A} 1, \mathrm{C} 4 / \mathrm{A} 1, \mathrm{C} 3 / \mathrm{A} 2$ e C4/A2), não foi diferente em todas as modalidades estudadas.

Verifica-se que houve pequena diminuição de amplitude $\mathrm{Na}-\mathrm{Pa}$ na reavaliação em ambos os gêneros. Em relação às latências das ondas $\mathrm{Na}$ e $\mathrm{Pa}$, verificou-se pequeno aumento na reavaliação em ambos os gêneros. Com relação à latência da onda $\mathrm{Pa}$, observou-se pequeno aumento na reavaliação para as modalidades C4/A1, C3/A2 e C4/A2 em ambos os gêneros (Tabela 2).

Comparando-se os resultados do P300 obtidos na avaliação e reavaliação, pode-se verificar ausência de diferença para a latência em ambos os gêneros ( $\mathrm{p}=0,49 \mathrm{e} 0,12$, respectivamente feminino e masculino). No entanto, para a amplitude N2-P3, observou-se, para ambos os gêneros, diferenças ( $\mathrm{p}=0,03$ e 0,003 , respectivamente).

De acordo com a Tabela 3, pode-se verificar aumento de latência na reavaliação para o gênero masculino e diminuição de latência na reavaliação para o gênero feminino. Para os valores obtidos na avaliação e reavaliação da amplitude N2-P3, houve aumento de amplitude na reavaliação para o gênero masculino e diminuição de amplitude na reavaliação para o gênero feminino.

Tabela 2. Diferença entre os valores da amplitude Na-Pa e latências $\mathrm{Na}$ e Pa nas modalidades C3/A1, C3/A2, C4/A1 e C4/A2 obtidos na avaliação e reavaliação nos gêneros feminino e masculino

\begin{tabular}{|c|c|c|c|c|c|c|}
\hline PEAML & Gênero & Modalidades & $\mathrm{N}$ & Média & Mediana & DP \\
\hline \multirow{8}{*}{$\begin{array}{l}\text { Amplitude } \mathrm{Na}-\mathrm{Pa} \\
(\mathrm{em} \mu \mathrm{V})\end{array}$} & \multirow{4}{*}{$\mathrm{F}$} & $\mathrm{C} 3 / \mathrm{A} 1$ & 25 & $-0,33$ & 0,08 & 1,68 \\
\hline & & C4/A1 & 25 & $-0,1$ & 0,35 & 1,34 \\
\hline & & $\mathrm{C} 3 / \mathrm{A} 2$ & 25 & $-0,91$ & $-0,4$ & 2,86 \\
\hline & & C4/A2 & 25 & $-0,24$ & $-0,17$ & 1,13 \\
\hline & \multirow{4}{*}{ M } & $\mathrm{C} 3 / \mathrm{A} 1$ & 24 & 0,14 & 0,21 & 3,4 \\
\hline & & C4/A1 & 24 & $-0,97$ & $-0,06$ & 2,86 \\
\hline & & $\mathrm{C} 3 / \mathrm{A} 2$ & 24 & $-0,45$ & $-0,59$ & 3,45 \\
\hline & & $\mathrm{C} 4 / \mathrm{A} 2$ & 24 & $-0,8$ & 0,37 & 3,67 \\
\hline \multirow{8}{*}{$\begin{array}{l}\text { Latência } \mathrm{Na} \\
\text { (em ms) }\end{array}$} & \multirow{4}{*}{$\mathrm{F}$} & $\mathrm{C} 3 / \mathrm{A} 1$ & 25 & 0,33 & 0,39 & 3,11 \\
\hline & & $\mathrm{C} 4 / \mathrm{A} 1$ & 25 & 0,62 & 0,39 & 3,12 \\
\hline & & C3/A2 & 25 & 0,48 & 0,39 & 1,43 \\
\hline & & C4/A2 & 25 & $-0,2$ & 0 & 3,02 \\
\hline & \multirow{4}{*}{ M } & $\mathrm{C} 3 / \mathrm{A} 1$ & 24 & 0,21 & 0,39 & 3,15 \\
\hline & & C4/A1 & 24 & 0,23 & 0 & 3,07 \\
\hline & & C3/A2 & 24 & 0,31 & 0,39 & 2,68 \\
\hline & & $\mathrm{C} 4 / \mathrm{A} 2$ & 24 & 0,21 & 0,59 & 1,96 \\
\hline \multirow{8}{*}{$\begin{array}{l}\text { Latência Pa } \\
\text { (em ms) }\end{array}$} & \multirow{4}{*}{$\mathrm{F}$} & $\mathrm{C} 3 / \mathrm{A} 1$ & 25 & $-0,51$ & $-0,39$ & 2,55 \\
\hline & & C4/A1 & 25 & 0,9 & 0,39 & 3,15 \\
\hline & & $\mathrm{C} 3 / \mathrm{A} 2$ & 25 & 0,98 & 0,78 & 2,62 \\
\hline & & $\mathrm{C} 4 / \mathrm{A} 2$ & 25 & 1,36 & 0,78 & 4,47 \\
\hline & \multirow{4}{*}{ M } & $\mathrm{C} 3 / \mathrm{A} 1$ & 24 & $-0,88$ & $-0,59$ & 4,93 \\
\hline & & $\mathrm{C} 4 / \mathrm{A} 1$ & 24 & 0,98 & 0,78 & 3,31 \\
\hline & & $\mathrm{C} 3 / \mathrm{A} 2$ & 24 & 0,33 & 0,59 & 4,36 \\
\hline & & C4/A2 & 24 & 1,01 & 0,2 & 4,2 \\
\hline
\end{tabular}

Legenda: $\mathrm{PEAML}=$ potencial evocado auditivo de média latência; $\mathrm{N}=$ número de indivíduos; $\mathrm{M}=\mathrm{masculino} ; \mathrm{F}=$ feminino; $\mathrm{ms}=$ milissegundos; $\mu \mathrm{V}=\mathrm{microvolts}$; DP = desvio-padrão 
Tabela 3. Diferença entre os valores da latência da onda P300 e amplitude N2-P3 obtidos na avaliação e reavaliação nos gêneros feminino e masculino

\begin{tabular}{lccccc}
\hline P300 & Gênero & $\mathrm{N}$ & Média & Mediana & DP \\
\hline Latência P300 & $\mathrm{F}$ & 50 & $-2,76$ & $-3,5$ & 27,99 \\
(em ms) & $\mathrm{M}$ & 48 & 6,54 & 5 & 29 \\
\hline Amplitude N2-P3 & $\mathrm{F}$ & 50 & $-2,68$ & $-2,33$ & 8,55 \\
(em $\mu \mathrm{V})$ & $\mathrm{M}$ & 48 & 6,26 & 7,21 & 13,25 \\
\hline
\end{tabular}

Legenda: $\mathrm{N}=$ número de orelhas; $\mathrm{M}=$ masculino; $\mathrm{F}=$ feminino; $\mathrm{ms}=$ milissegundos; $\mu \mathrm{V}=$ microvolts; $\mathrm{DP}=$ desvio-padrão

\section{DISCUSSÃO}

A estabilidade das variáveis estudadas nos PEA tem sido investigada, tendo em vista o crescente uso destas medidas na identificação e no monitoramento da evolução de comprometimentos na via auditiva central.

O PEATE é o potencial evocado auditivo mais utilizado na prática clínica e suas aplicações clínicas no monitoramento da via auditiva têm sido discutidas desde a década de 70 . Autores referiram que suas medidas demonstraram ser bons preditores da função auditiva após neurocirurgias, embora não tenham fornecido informações importantes com relação à condição neurológica do paciente ${ }^{(15,16)}$.

Na presente pesquisa, com relação ao PEATE, verificou-se estabilidade das variáveis estudadas no período de três meses, visto que não foi observada diferença para as latências absolutas das ondas I, III e V e interpicos I-III, III-V e I-V, em ambos os gêneros. Tais achados corroboraram os de Bergamaschi et al. ${ }^{(17)}$ que observaram estabilidade dos componentes do PEATE em adultos após sete dias. Os resultados de um estudo que avaliou e comparou a estabilidade do PEATE em crianças e adultos, indicaram que o grupo das crianças na faixa etária entre dez e 12 anos apresentou valores de latências estáveis, semelhantes aos de adultos ${ }^{(18)}$. Da mesma forma, verificou-se confiabilidade teste-reteste satisfatória em pesquisa realizada com seis indivíduos, num intervalo inter-sessões de seis meses ${ }^{(19)}$.

O PEAML é considerado um dos melhores testes para se avaliar o sistema nervoso auditivo central, além de ser reconhecidamente útil no monitoramento do processo terapêutico. Nossos resultados indicaram estabilidade dos resultados do PEAML obtidos na avaliação e reavaliação após o período de três meses (amplitude Na-Pa e latência das ondas $\mathrm{Na}$ e $\mathrm{Pa})$, corroborando resultados encontrados ${ }^{(20)}$. Nesses estudos, foram realizadas gravações seqüenciais dos potenciais de média latência em indivíduos normais num período de dois anos e meio, e verificou-se estabilidade das latências de todos os componentes. Da mesma forma, pesquisadores ${ }^{(21)}$ avaliaram indivíduos adultos por meio dos potenciais evocados auditivos de curta, média e longa latências, e verificaram a estabilidade destes potenciais no teste-reteste. Constataram ainda que a magnitude da estabilidade das medidas de amplitude foi menor quando comparada às medidas de latência.

Com relação aos parâmetros do P300, observou-se estabilidade no parâmetro latência; no entanto, a amplitude N2-P3 não foi estável durante o período de três meses, corroborando a idéia de que este componente apresenta grande variabilidade, o que contra-indica sua utilização no monitoramento terapêutico. De fato, autores referem que a amplitude N2-P3 é uma variável pouco estudada por apresentar variabilidade intra-sujeitos e não possuir critérios de normalidade bem estabelecidos ${ }^{(14)}$. Por outro lado, autores, ao avaliar indivíduos adultos saudáveis por meio dos Potenciais Evocados Auditivos de Longa Latência (PEALL), referiram alta confiabilidade quando comparados os resultados obtidos no teste e reteste, das amplitudes dos componentes N1, MMN, P3 e N4 ${ }^{(7)}$.

Diversos estudos têm indicado que a latência do P300 apresenta confiabilidade teste-reteste em indivíduos adultos normais $^{(5,22,23)}$. Autores ${ }^{(23)}$ observaram confiabilidade dos componentes do P300 intra e entre sessões, sugerindo que tais padrões observados em longo-termo podem refletir habituação ou desabituação de determinados processos no sistema nervoso central. Grande confiabilidade nos dados obtidos intra-sujeitos com relação à onda $\mathrm{P} 3$ foi observada no estudo da estabilidade dos potenciais evocados auditivos em indivíduos idosos. Isso indica sua importância na detecção de mudanças pré-clínicas nesta população $0^{(5)}$. Pesquisadores ${ }^{(24)}$ estudaram a replicabilidade do Mismatch Negativity (MMN), potencial de longa latência endógeno, em 15 adultos saudáveis, e demonstraram que este potencial demonstrou estabilidade quando se comparou os resultados obtidos na avaliação e reavaliação.

Alterações no P300 sugerem a presença de comprometimentos em regiões corticais da via auditiva, relacionadas às habilidades como processamentos cognitivos relacionados à memória, atenção e discriminação auditiva. Conseqüentemente, a diminuição da latência da onda P300 está relacionada ao aumento da capacidade cognitiva ${ }^{(25,26)}$. Portanto, efetuar o seguimento de indivíduos com doenças que comprometem a capacidade cognitiva por meio do $\mathrm{P} 300$ pode trazer benefícios, pois essa medida eletrofisiológica pode fornecer informações a cerca de mudanças comportamentais que se manifestam mais tardiamente.

Os resultados da presente pesquisa reforçam a idéia do uso dos potenciais evocados auditivos como ferramenta terapêutica no monitoramento da evolução da reabilitação fonoaudiológica e treinamento auditivo ${ }^{(27)}$, tanto em adultos como crianças, pois podem fornecer medidas eletrofisiológicas quantitativas e objetivas a cerca da plasticidade neuronal decorrente da estimulação auditiva. São necessários estudos adicionais, que investiguem a estabilidade dos potenciais evocados auditivos, para que estas variáveis fiquem bem definidas.

\section{CONCLUSÃO}

Observou-se estabilidade das variáveis estudadas no PEATE, PEAML em adultos normais após um período de três meses. Com relação ao P300, observou-se estabilidade da latência da onda P300, contudo, tal estabilidade não foi observada na amplitude N2-P3

\section{AGRADECIMENTOS}

Agradecemos à Fundação de Amparo à Pesquisa do Estado de São Paulo (FAPESP), pelo apoio concedido para realização dessa pesquisa, sob processo número 07/58595. 


\begin{abstract}
Purpose: To evaluate the stability of parameters of auditory evoked potentials in normal adults. Methods: Forty-nine normal subjects with ages from 18 to 40 years ( 25 females and 24 males) were submitted to audiological and electrophysiological hearing evaluation (auditory brainstem response - ABR, middle latency response - MLR, and cognitive potential - P300). Subjects were reassessed three months after the initial evaluation. Results: Significant differences were observed between genders regarding the wave latencies III and V and the interpeaks I-III and I-IV of ABR, and the amplitude N2-P3 of the P300. No differences were found between the results of initial and final assessments for the parameters of the ABR, MLR (Na, Pa latencies and Na-Pa amplitude) and P300 (P300 latency). Conclusion: Except for the N2-P3 amplitude, it was observed stability of the parameters of ABR, MLR and P300 in normal adults after a period of three months.
\end{abstract}

Keywords: Hearing tests; Hearing; Evoked potentials, auditory; Event-related potentials, P300; Adult

\title{
REFERÊNCIAS
}

1. Picton TW, Hillyard SA, Krausz HI, Galambos R. Human auditory evoked potentials. I. Evaluation of components. Electroencephalogr Clin Neurophysiol. 1974;36(2):179-90.

2. Musiek FE, Berger BE. How electrophysiologic tests of central auditory processing influence management. In: Bess FV, organizador. Children with hearing impairment. Nashville: Bill Wilkerson Center Press; 1998. p. $145-62$.

3. Andrade CRF, Cunha MC, Matas CG. Gagueira: aspectos auditivos, neuromotores, psíquicos e de linguagem. In: XIV Congresso Brasileiro de Fonoaudiologia, 2006, Salvador.

4. Pekkonen E, Rinne T, Näätänen R. Variability and replicability of the mismatch negativity. Electroencephalogr Clin Neurophysiol. 1995;96(6):546-54.

5. Sandman CA, Petterson JV. The auditory event-related potential is a stable and reliable measure in elderly subjects over a 3 year period. Clin Neurophysiol. 2000;111(8):1427-37. Comment in: Clin Neurophysiol. 2000;111(8):1425-6.

6. Walhovd KB, Fjell AM. One-year test-retest reliability of auditory ERPs in young and old adults. Int J Psychophysiol. 2002;46(1):29-40.

7. Lew HL, Gray M, Poole JH. Temporal stability of auditory event-related potentials in healthy individuals and patients with traumatic brain injury. J Clin Neurophysiol. 2007;24(5):392-7.

8. Santos TMM, Russo ICP. A prática da audiologia clínica. São Paulo: Cortez; 1986. p. 81-98.

9. Jerger J. Clinical experience with impedance audiometry. Arch Otolaryngol. 1970;92(4):311-24.

10. Carvallo RMM, Carvalho M, Ishida IM. Auditory profile in individuals with and without CAPD. In: American Academy of Audiology Annual Convention \& Exposition, 12th., São Paulo: 2000. São Paulo; 2000. p.195.

11. Lloyd LL, Kaplan H. Audiometric interpretation: a manual of basic audiometry. Baltimore: University Park Press; c1978.

12. Gates GA, Chakeres DW. Interpretation of diagnostic tests for acoustic neuroma. 3rd ed. Rochester: American Academy of Otolaryngology-Head and Neck Surgery Foundation; 1988.

13. Durrant JD, Ferraro JA. Potenciais auditivos evocados de curta latência: eletrococleografia e audiometria de tronco encefálico. In: Musiek FE, Rintelmann WF. Perspectivas atuais em avaliação auditiva. Barueri: Manole; 2001. p.193-238.
14. Musiek FE, Lee WW. Potenciais auditivos de média e longa latência. In: Musiek FE, Rintelmann WF. Perspectivas atuais em avaliação auditiva. Barueri: Manole; 2001. p. 239-67.

15. Starr A. Auditory brain-stem responses in brain death. Brain. 1976; 99(3):543-54.

16. Fischer C, Ibañez V, Mauquiere F. [Peroperative monitoring of early auditory evoked potentials]. Presse Med. 1985;14(37):1914-8. French.

17. Bergamaschi R, Romani A, Versino M, Sartori I, Callieco R, Cosi V. A one- week test-retest reliability study of Brainstem Auditory Evoked Potentials. Boll Soc Ital Biol Sper. 1992;68(7):467-74.

18. Lauter JL, Oyler RF. Latency stability of auditory brainstem responses in children aged 10-12 years compared with younger children and adults. Br J Audiol. 1992;26(4):245-53.

19. Rosenhamer HJ, Lindström B, Lundborg T. On the use of click-evoked electric brainstem responses in audiological diagnosis. I. The variability of the normal response. Scand Audiol. 1978;7(4):193-205.

20. Robinson K, Rudge P. The stability of the auditory evoked potentials in normal man and patients with multiple sclerosis. J Neurol Sci. 1978;36(1):147-56.

21. Lauter JL, Karzon RG. Individual differences in auditory electric responses: comparisons of between-subject and within-subject variability. V. Amplitude-variability comparisons in early, middle, and late responses. Scand Audiol. 1990;19(4):201-6.

22. Sklare DA, Lynn GE. Latency of the P3 event-related potential: normative aspects and within-subject variability. Electroencephalogr Clin Neurophysiol. 1984;59(5):420-4.

23. Polich J, Howard L, Starr A. P300 latency correlates with digit span. Psychophysiology. 1983;20(6):665-9.

24. Picton TW. The P300 wave of the human event-related potential. J Clin Neurophysiol. 1992;9(4):456-79.

25. Tervaniemi M, Sinkkonen J, Virtanen J, Kallio J, Ilmoniemi RJ, Salonen $\mathrm{O}$, Näätänen R. Test-retest stability of the magnetic mismatch response (MMNM). Clin Neurophysiol. 2005;116(8):1897-905.

26. Raikkonen K, Birkás E, Horváth J, Gervai J, Winkler L. Test- retest reliability os auditory ERP components in healthy 6-year-old children. Neuroreport. 2003;14(16):121-5.

27. Kinoshita S, Inoue M, Maeda H, Nakamura J, Morita K. Long-term patterns of change in ERPs across repeated measurements. Physiol Behav. 1996;60(4):1087-92. 